\title{
Automated 3-D tracking of video-captured movement using the example of an aquatic mollusk
}

\author{
J. F. DERRY and C. J. H. ELUIOTT \\ University of York, York, England
}

\begin{abstract}
We describe a computer program that tracks the three-dimensional coordinates of a snail (moving within a tank) from images captured by a single video camera. The tank and a mirror placed at $45^{\circ}$ to one side are viewed to provide direct and reflected images of a pond snail, Lymnaea stagnalis, moving around the tank. The output of the camera is captured with an IBM-compatible PC using a framegrabbing VideoBlaster board. The main features of our Windows program is the frame-by-frame analysis of the captured video, enhancement of contrast, location of dark "snails," discrimination by size, trigonometric determination of the $x, y, z$ coordinates, and the final export of data in a spreadsheetready format. After the user has outlined the position of the tank on the screen and set the discrimination thresholds, no further user activity is required. This makes the analysis of slowly moving mollusks much less operator intensive and less tedious. The method is applicable to other faster moving organisms (e.g., fish) because the analysis is performed on captured video sequences.
\end{abstract}

Many organisms can move freely in air or water. To study their locomotory behavior, it is necessary to track their position in all three coordinates, $x, y$, and $z$. A single image is normally insufficient to obtain this information because such an image is only two dimensional. However, the three-dimensional (3-D) position can be calculated from two images of the tank seen from separate viewing points. One way to obtain the second image is to place a mirror to the side of the tank (see Figure 1). The $x, y, z$ coordinates can then be calculated using nearfield photogrammetry (Pereira \& Oliveira, 1994). If the position of the organism in the two views is digitized manually, analysis of a slowly moving mollusk becomes tedious when long periods of study are required, because it requires extensive human intervention. We report here a computer program that identifies the dark images of a snail and then uses the photogrammetry calculations of Pereira and Oliveira (1994) to calculate its 3-D position while it moves freely without restraint in its tank. Only during the initial setup is operator involvement required.

\section{METHOD}

\section{Animals}

A pond snail, Lymnaea stagnalis, was kept in a tank of water and fed lettuce as required. The snail moved freely over the sides and through the water.

\section{Tank}

The tank had a square base of side $300 \mathrm{~mm}$ and was filled with standard snail water (Elliott \& Benjamin, 1989)

The authors would like to thank David White for reading the manuscript. Correspondence should be addressed to C. J. H. Elliott, Department of Biology, University of York, P.O. Box 373, York YO1 5YW, England (e-mail: cje2@york.ac.uk). to about $250 \mathrm{~mm}$ in depth. The back, floor, and one side were constructed of white Perspex; the front and other side were constructed of transparent Perspex. On the transparent side, a mirror was placed, angled at $45^{\circ}$ to the side of the tank. Thus, when the tank was viewed from the front, two images were seen, the direct and mirror images. The tank was illuminated with a light placed above it, so that the illumination of the white Perspex was as even as possible. Lymnaea stagnalis are normally dark brown or black and contrast well with the white sides of the tank.

\section{Video Capture}

A black-and-white video camera was placed in the front of the tank and aligned so that the transparent edge of the tank was exactly on the axis of the camera. In our experiments, the distance between camera and tank was $1,130 \mathrm{~mm}$, which meant that the tank plus its image in the mirror (the virtual tank) occupied the full frame width (see Figure 2). The output from the video camera was applied to a Creative Labs VideoBlaster board, and the video was recorded on the hard disk of an IBM-compatible PC with a 33- $\mathrm{MHz} 486$ processor in AVI format using the VidCap program (part of Microsoft Video for Windows 1.1 and supplied with the VideoBlaster and other video acquisition cards). In our experiments, frames were taken once every $10 \mathrm{sec}$, the slowest rate possible with the VidCap program. However, because our analysis is made directly from captured video footage, our program would be equally suitable for AVI-format sequences captured at faster rates (e.g., of fish swimming).

\section{Program Construction}

The program was constructed in three linked modules: (1) outlining the tank and setting the thresholds, (2) discrimination of direct and mirror images of the snail, and (3) trigonometric calculations. The only operator inter- 


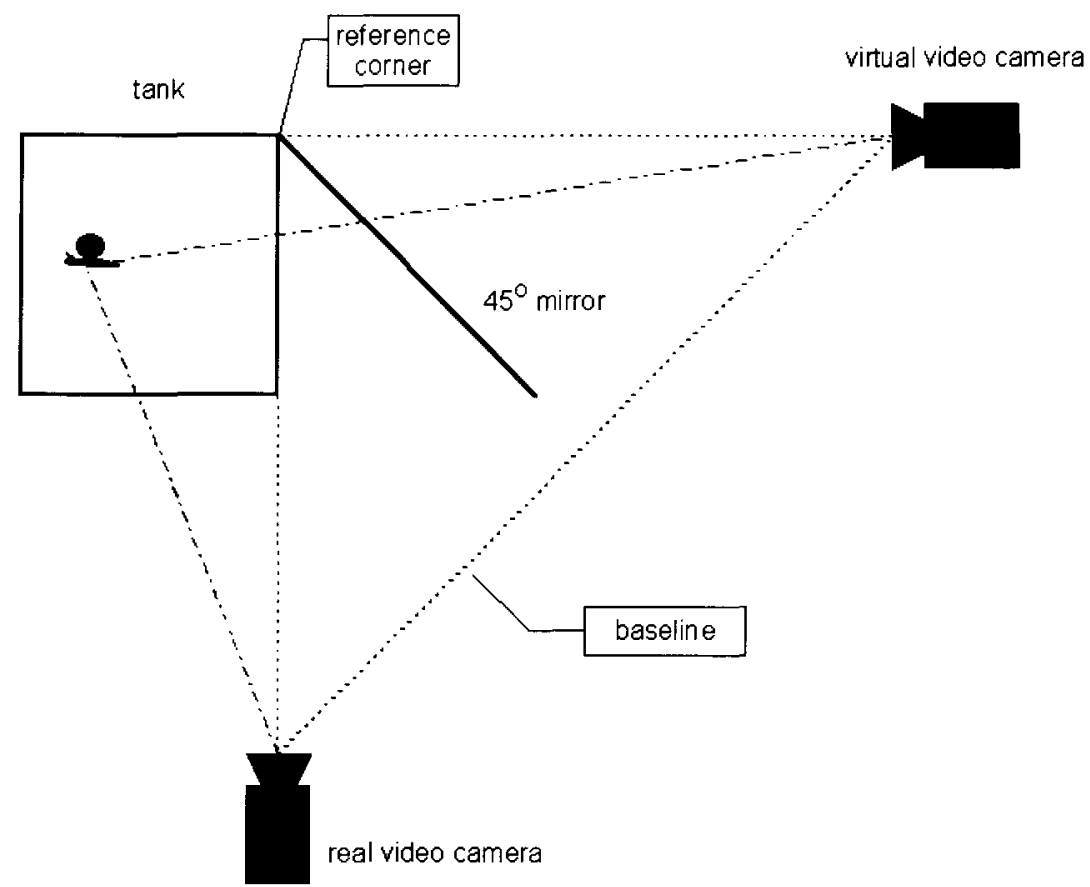

Figure 1. The experimental setup as viewed from above. The snail moves freely in the waterfilled tank. The mirror is placed at $45^{\circ}$ to the side of the tank. A single video camera, whose output is passed to a VideoBlaster board, is used to record both the direct view and the mirror view of the tank. The mirror provides a "virtual" camera apparently situated to the side of the tank. The real camera, virtual camera, and reference corner of the tank form a triangle (dotted lines), whose baseline is calculated in our program from the tank-camera distance and the angular position of the reference corner in the first video frame. A second triangle (dash/dotted line) is formed by the snail and real and virtual cameras. In successive frames, the location of the snail is found from its angular position and the calculated baseline (Pereira \& Oliveira, 1994).

vention is required in Part 1 while the location of the tank within the video frame and the threshold criteria for snail identification are set. The program was devised for the Windows 3.1 environment and uses the Video for Windows run-time library.

\section{Outlining the Tank and Setting the Thresholds}

Once an AVI-format file has been opened, it is necessary to define the position of the direct and mirror views of the tank on the initial video frame. The user chooses a Windows dialog box to indicate whether (s)he wishes to outline the front or the mirror view and then drags the mouse across the screen to mark the outline of the tank (see Figure 2). In the mirror view, the tank does not appear rectangular but trapezoidal (because the front is closer and hence appears larger than the back), and so the user can adjust the position of each corner of the trapezium exactly to fit this perspective. (If the outlines do not match exactly, the operator can redo the outlining process.) A second Windows dialog box is used to set the threshold criteria for identification of a snail, with thresholds for size and light intensity (the thresholds for the red, green, and blue components can be set independently).

\section{Discrimination of the Snail's Images}

A snail is the only large dark object in the tank, so the two criteria set above are sufficient to discriminate the images of a snail. The program proceeds as follows: Each frame of the AVI is copied to a workspace window. In this copy, the border zones around the tank and its mirror view are set white. (This prevents objects outside the tank being misidentified as "snails.") The contrast is then enhanced: the 256 shades of gray present in the copy of the video frame are dithered to two shades (black and white) using the intensity threshold chosen earlier (see Figure 3 for an example scan). This process leaves the snail and any feces black but the rest of the tank (including lettuce leaflets) white. The resulting two-tone picture is then scanned a second time, measuring the circumference of each object found so that the feces (which are small) are rejected; large snails are accepted. The center of the accepted target is passed to the trigonometric analysis module.

\section{Trigonometric Calculations}

Standard near-field photogrammetry equations (Pereira \& Oliveira, 1994) are used to calculate the $x, y, z$ coordinates from the direct and mirror positions reported 


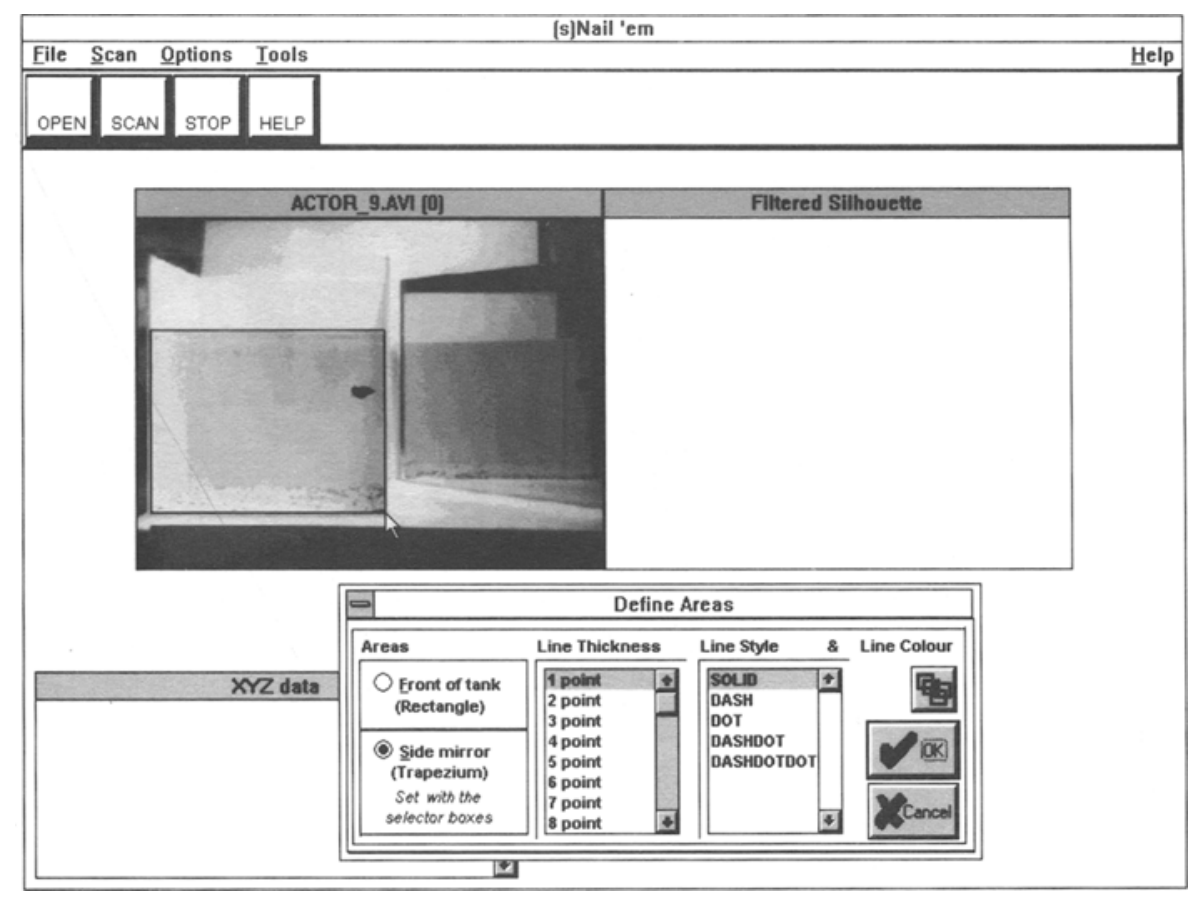

Figure 2. Outlining the tank: A selected screen view during the outlining process. In the AVI window, the direct and mirror views of the tank can be seen. The outline of the direct view is already drawn by the operator. The dialog box for outlining the mirror view has been evoked from the menu. When the operator clicks $O K$, (s)he will be able to define the mirror view of the tank.

by the scanning process. The corner of the tank and the real and "virtual" positions of the camera form a triangle (see Figure 1). The baseline between real and virtual cameras can be calculated from the tank-camera distance and the angles subtended by the tank corner in direct and mirror views. A second triangle with the same baseline is formed by the "snail" and the real and virtual positions of the camera. The angles subtended by the snail in the two views (together with the calculated baseline) provide sufficient information to calculate the $x, y, z$ coordinates. The equations used by Pereira and Oliveira (1994) to calculate the position were rearranged using the computer algebra package Maple and the resulting $C$ code incorporated into the program. The one unknown variable in their equations, the value of the focal length $(f)$ in pixels was derived from the pixel coordinates of the tank captured during the outlining process. At the end of the scan of each frame, the pixel coordinates of the "snails" in direct and mirror views are used to calculate the true $x, y, z$ position of the snail in the tank. The $x, y, z$ coordinates of the detected snail images are shown in a Windows list box, together with its direct and mirror sizes, and the current video frame number. After processing the last frame, the operator saves the data under a suitable file name. The data are exported in a spreadsheetready format, which can be read by SPSS, SigmaPlot, Minitab, and other packages. Figure 4 shows the movements of a snail over a period of $5 \mathrm{~min}$.

\section{DISCUSSION}

The main advance that we report is the $x, y, z$ localization of slowly moving molluskan targets without the need for prolonged operator intervention. The discrimination criteria of size and blackness are sufficient to distinguish black snails in the white tank and also apply for other species (e.g., fish, insects) that can be observed in a suitable tank with front and side views. The method is not confined to a particular size of tank nor to a particular camera-object distance. In our experiments, the AVI files were recorded from a black-and-white video; however, if a color camera were used, then snails marked with different colors could be distinguished by setting different color thresholds on different runs of our analysis program.

Furthermore, because the analysis is made on AVIformat video recorded on the hard disk of the computer, it is not restricted to particular speeds of movement or to particular camera/frame grabber technology. With the slowly moving mollusk, Lymnaea stagnalis, analysis of videos recorded at $50 \mathrm{sec} /$ frame provides sufficient data points, but more rapid movements can be analyzed by using faster frame rates. On our $33-\mathrm{MHz} 486$, the VideoBlaster will capture frames at $0.1-\mathrm{sec}$ intervals; faster PCs claim to reach $0.04 \mathrm{sec} /$ frame. Though faster frame rates improve temporal resolution, they also increase disk usage (at $1 \mathrm{sec} /$ frame about $3.5 \mathrm{MB}$ will be used each minute of video); therefore, some compromise may be needed 
(a)

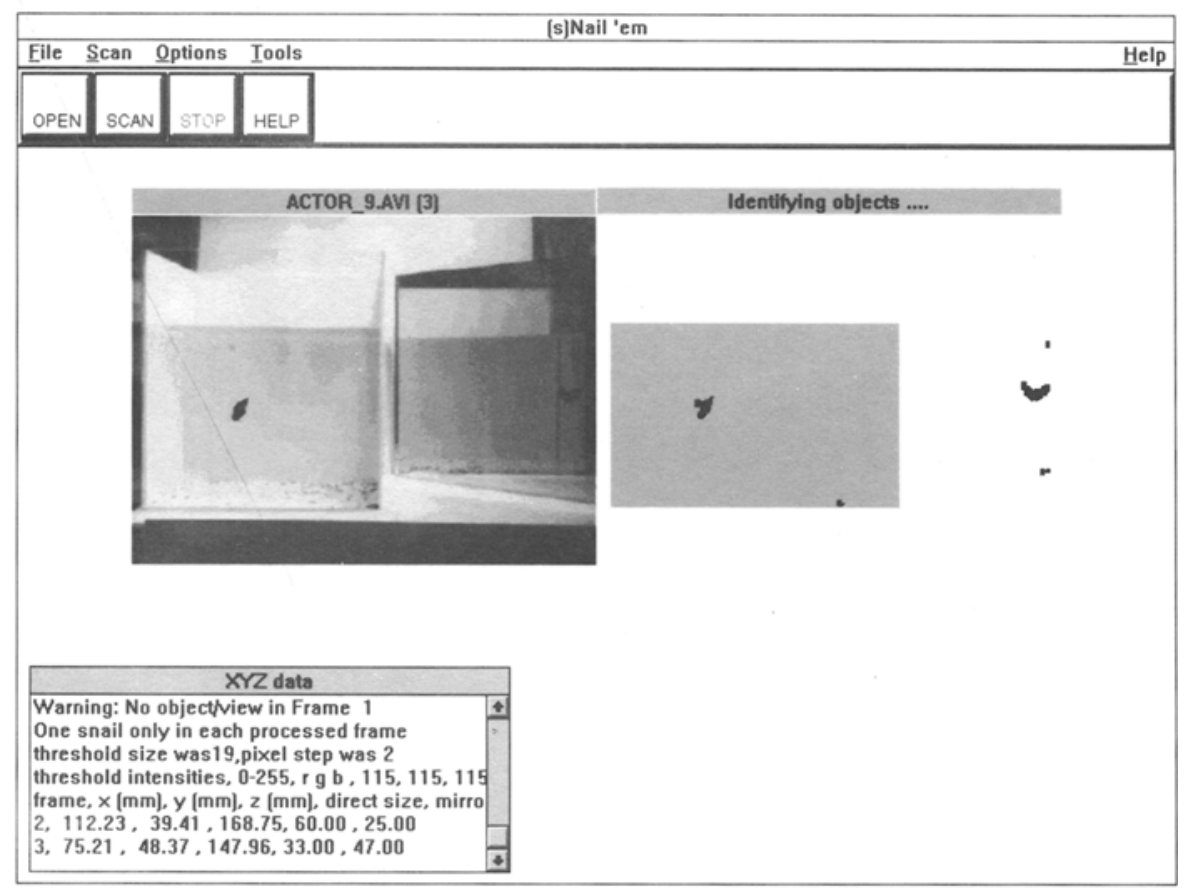

(b)

(c)
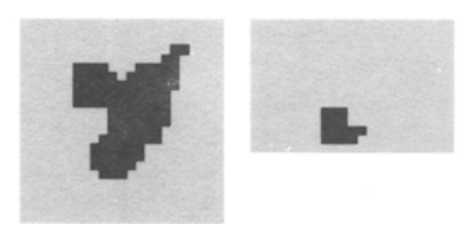

Figure 3. Scanning the tank. (a) A "screen shot" showing the scanning process. The direct and mirror images of the tank have been copied to the right hand (workspace window) and then surrounded by a white border to exclude objects outside the tank. Second, the contrast has been increased so that the snail and some feces are turned black. Now, a third phase of the scan is under way, in which objects, discriminated on the base of size, are identified and their 3-D position calculated. As points on the screen are examined for the presence of an object, the pixels are turned gray. When the direct image of the snail was encountered it was left black (see detail view $b$ ), and the feces were rejected as too small (shown dark gray in detail view c). At the end of the scan, the 3-D position will be calculated. The XYZ listbox (bottom left) shows the $x, y, z$ coordinates determined from the previous frames.

between the temporal resolution and the total duration of the recording.

Two possible sources of error are discrimination of the correct target and accuracy of the camera/tank setup. We find that the system is both accurate and versatile enough to cope with variation in different targets.

\section{Target Discrimination}

The main problem in distinguishing snails results from uneven illumination of the tank, with errors occurring if the back and sides of the tank are inconsistently bright. Occasional problems with shadows cast by lettuce leaflets were eliminated by use of a $60-\mathrm{W}$ light about $1 \mathrm{~m}$ above the tank. This also reduces the number of multiple reflections that give rise to false-positive images, in which a single snail gives more than one image per view of the tank. Warnings about this problem are placed at the head of the listbox and in the datafile, together with the errant frame numbers, so that the discrimination thresholds may be adjusted.

A second discrimination error is failure to find the snail in a particular view of the tank. These false negatives usually result from incorrect threshold settings, and this is again reported at the head of the listbox and the output data file. The ability to set the discrimination thresholds for either all or part of a video sequence means that our approach is highly versatile and can cope with different sizes of "snails" in the tank. 


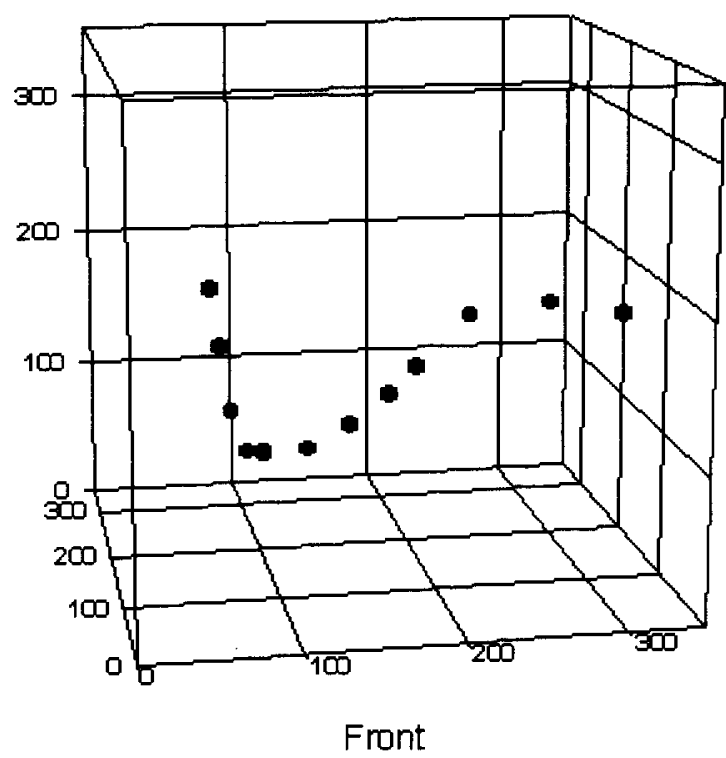

Figure 4. A drawing from Jandel Corporation's Sigmaplot showing the motion of a snail over 12 frames of an AVI. The frames were grabbed at 10 -sec intervals, and selected frames were then removed from the resultant AVI file to produce a rate of $50 \mathrm{sec} /$ frame.

\section{Accuracy of the Method}

We have used AVI-format files captured by the VideoBlaster with a resolution of $320 \times 240$ pixels. As the AVI shows direct and mirror views, the front and side views of the tank occupy about 150 pixels, so the possible resolution is just over $1 \%$. For our $300 \times 300 \mathrm{~mm}$ tank, this represents an error of $3 \mathrm{~mm}$, much less than the size of the snail, $10-15 \mathrm{~mm}$ long. A bigger source of error is likely to be the inaccuracies in measurement of the focal length and object-camera distances, in aligning the mirror at $45^{\circ}$, or in setting up the camera normal to the front of the tank-all of which lead to errors in the trigonometric calculation. Angular errors lead to rhomboid rather than square base of the tank. With distance errors, the apparent size of the tank is changed, but its orientation does not alter significantly. Other errors might be expected from refraction of light at the tank/water interface; however, these will be consistent for any given tank. Our system has an error comparable to the manual calculations of Pereira and Oliveira (1994), which were given at $3 \%-5 \%$. For videos of any one tank, however, our method will give consistent results because human operator errors in clicking on targets have been eliminated.

\section{Conclusion}

The program described here provides an automated method to study the locomotory behavior of mollusks. It reduces the tedium significantly. It is simple to use, but the ability to adjust the thresholds to discriminate the "snails" means that it is versatile and can be adjusted for other animals within a tank.

\section{Availability}

The executable and necessary supporting software is available free of charge. It requires Windows 3.1 running on a monitor with at least $800 \times 600$ pixels. It can be obtained using Netscape or a similar Web browser. The URL http://www.york.ac.uk/ cje2/snails has instructions for downloading the executable file and necessary supporting software. It also has a link to obtain the Video for Windows run-time library. The software includes a detailed Help file in the Windows Help format: This has full instructions for setting up the files correctly and running a test video, which is also supplied. The software package contains the Maple (and derived C) algorithms for calculating the 3-D position from the pixel coordinates. The original source code may be obtained by e-mail contact with the authors.

\section{REFERENCES}

Elliott, C. J. H., \& Benjamin, P. R. (1989). Esophageal mechanoreceptors in the feeding system of the pond snail, Lymnaea stagnalis. Journal of Neurophysiology, 61, 727-736.

Pereira, P., \& Oliveira, R. F. (1994). A simple method using a single video camera to determine the three-dimensional position of a fish. Behavior Research Methods, Instruments, \& Computers, 26, 443-446.

(Manuscript received February 26, 1996; revision accepted for publication June 20,1996.) 